\title{
Belgeo
}

Revue belge de géographie

$4 \mid 2006$

Miscellaneous

\section{Géographie mondiale des alliances de compagnies aériennes}

A world geography of airlines alliances

\section{Mathieu Strale}

\section{(2) OpenEdition}

\section{Journals}

\section{Édition électronique}

URL : http://journals.openedition.org/belgeo/11761

DOI : 10.4000/belgeo.11761

ISSN : 2294-9135

Éditeur :

National Committee of Geography of Belgium, Société Royale Belge de Géographie

\section{Édition imprimée}

Date de publication : 31 décembre 2006

Pagination : 437-464

ISSN : 1377-2368

\section{Référence électronique}

Mathieu Strale, « Géographie mondiale des alliances de compagnies aériennes », Belgeo [En ligne]

4 | 2006, mis en ligne le 31 octobre 2013, consulté le 19 avril 2019. URL : http://

journals.openedition.org/belgeo/11761; DOI : 10.4000/belgeo.11761

Ce document a été généré automatiquement le 19 avril 2019.

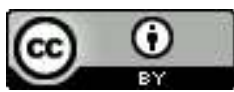

Belgeo est mis à disposition selon les termes de la licence Creative Commons Attribution 4.0 International. 


\title{
Géographie mondiale des alliances de compagnies aériennes
}

\author{
A world geography of airlines alliances
}

\author{
Mathieu Strale
}

\section{Les alliances de compagnies aériennes, un phénomène récent}

1 Suite à la libéralisation progressive du transport aérien, les compagnies aériennes ont été confrontées à deux défis de taille : la réduction des coûts et la résistance à la concurrence des autres groupes. Les alliances de compagnies aériennes, nées à la fin des années 80 , constituent une réponse aux conditions économiques et politiques actuelles du transport aérien (OMC, 2003). Les compagnies ont en effet multiplié les accords entre elles, si bien que le nombre d'accords ou de coopérations commerciales est passé de 200 à 500 entre 1991 et 2001 (Kleymann, 2002).

2 Ces accords sont de différents types (Merlin, 2000):

- l'affrètement d'avions d'autres compagnies, surtout pour des vols locaux ;

- l'utilisation de vols de petites compagnies en franchise par d'autres compagnies, surtout pour des liaisons locales (Denton \& Dennis, 2000).

- le partage de codes par deux compagnies (ou plus) : le vol a plusieurs codes, un par compagnie. Les compagnies apposant leur code sans opérer le vol peuvent proposer des sièges à leurs clients sur des vols qu'elles ne réalisent pas, tandis que les compagnies transporteuses ont l'assurance de mieux remplir l'appareil et parfois d'affréter un plus gros avion, ce qui réduit les coûts par passager-km.

- les «joint ventures»: deux (ou plusieurs) compagnies mettent en commun une partie de leurs réseaux et de leurs revenus sur certaines routes ;

- la prise de participation financière de certaines compagnies dans d'autres, pouvant aller jusqu'à la fusion (par exemple Air France et KLM). 
- les alliances commerciales: les compagnies se regroupent sous une marque commune, mettent en commun leurs services de vente et leurs programmes de voyageurs fréquents et développent des partages de codes sur de nombreux vols (Gudmundsson et al., 2002). Star Alliance et Skyteam, lesquelles regroupent de nombreuses compagnies et couvrent près de la moitié des vols réguliers. Elles sont basées sur une alliance commerciale à laquelle s'ajoutent des « joint ventures », des participations financières, des fusions... peu d'analyses géographiques ont été réalisées sur le sujet, qui a surtout été étudié sous un angle économique, politique. L'impact géographique du transport aérien est pourtant fondamental en raison des répercussions sur la couverture des vols, sur la desserte des villes et des pays et sur l'insertion des régions dans l'économie mondiale. C'est un des aspects de la mondialisation de l'économie, qui implique des flux de plus en plus importants entre certains lieux et la formation de réseaux conditionnant l'insertion des régions dans les échanges. Cet article traitera de la géographie des alliances à l'échelle mondiale, à partir de la situation en 2005.

5 La première partie de l'article sera consacrée à la géographie, à l'échelle mondiale, des alliances de compagnies aériennes : il s'agira de déterminer les zones d'implantation des alliances, les lieux où leur offre est concentrée, et de tenter d'entrevoir leur évolution future. Ensuite, l'organisation du trafic des alliances, la répartition spatiale des compagnies qui les composent et les mesures mises en place pour couvrir l'offre mondiale de services aériens seront analysées. Enfin, en comparant la situation actuelle à celle qui prévalait dix ans auparavant, on tentera de mettre en évidence la stratégie générale de développement des alliances.

6 Les principales sources de notre recherche sont les bases de données OAG (Official Airline Guide) éditées en janvier 1995, 2000 et 2005. Elles regroupent pour les trois années les prévisions d'offre des compagnies aériennes du monde entier pour les douze mois à venir, à partir du premier janvier. Dans ce contexte-ci, seuls les vols réguliers de passagers seront analysés, laissant de côté le fret et les vols charters. Les données OAG sont compilées à partir des vols que les compagnies prévoient d'opérer durant les mois à venir. Leur exhaustivité et leur exactitude diminuent quand on s'éloigne du début de la période : les compagnies peuvent, en effet, modifier leur plan de vols en cours d'année, des compagnies peuvent disparaître ou entrer sur le marché... Pour limiter les risques d'erreurs, il est donc préférable de travailler sur la période la plus proche de la date d'édition de la base de données. Les traitements seront dès lors réalisés sur les données de janvier de 1995, 2000 et 2005.

\section{La formation des alliances des compagnies aériennes}

7 Le transport aérien est une économie très fragile, dont les marges bénéficiaires sont faibles et les risques et les coûts élevés. Pour subsister, il faut en principe avoir une offre la plus générale possible (Kleymann, 2002), mais le transport aérien demande des investissements très coûteux, ce qui rend impossible la couverture de l'ensemble du marché par une seule compagnie (Biplan, 2004). Secteur très sensible aux conditions économiques et politiques, le transport aérien a été frappé de plein fouet par la crise qui a suivi les événements du 11 septembre 2001 : les compagnies ont essuyé de fortes pertes. 
Elles ont alors rationalisé leur réseau long-courrier pour le concentrer plus encore sur quelques hubs et elles ont diminué le nombre de destinations desservies (Dennis, 2005).

La libéralisation progressive a donc changé les règles régissant le transport aérien, mais ce phénomène concerne surtout l'Union européenne et l'Amérique du nord, tandis qu'en dehors de ces régions, les liaisons internationales sont encore souvent régies par des accords bilatéraux entre pays (Biplan, 2004). De plus, l'accès du capital étranger à l'actionnariat des compagnies et l'accès au ciel national de compagnies étrangères sont encore souvent très limités, ce qui empêche la formation de compagnies mondiales (Oum et al., 2001). Certains marchés importants, tels que les vols intérieurs aux Etats-Unis, ne sont accessibles aux étrangers que via des alliances avec des compagnies locales, seules autorisées à y voler.

\section{Une réponse aux pressions du marché}

9 Une réponse actuelle des compagnies aériennes à ces contraintes est la spécialisation dans une niche, et la formation d'alliances entre compagnies spécialisées pour couvrir tout le marché. La survie à long terme des compagnies aériennes classiques semble conditionnée par leur affiliation à une alliance majeure (Agusdinata \& Klein, 2002). Plusieurs spécialistes de l'économie du transport aérien estiment que le mouvement aboutira à terme à la création de «méga-compagnies » mondiales (Gudmundsson \& Rhoades, 2001).

Les alliances peuvent être définies comme des structures dans lesquelles les acteurs cherchent, d'une part, à préserver leur indépendance et, d'autre part, à déléguer une partie de leur autorité pour former une alliance ayant une existence propre (Kleymann, 2002). Leur pouvoir est actuellement limité, car les compagnies restent responsables de leur réseau et de leurs finances. La fragilité des alliances oblige les compagnies à garder la possibilité d'être viables seules, et limite donc les liaisons qu'elles consentent à abandonner à leurs partenaires. Mais il existe un discours fédérateur au sein des alliances et l'on va progressivement vers plus d'intégration (Kleymann, 2002). La structure hiérarchique des alliances est à ce jour assez peu développée, même si elle commence à se mettre en place avec l'apparition des compagnies régionales ou affiliées chez Star Alliance et Skyteam, et la formation d'alliances à plusieurs étages hiérarchiques (Kleymann, 2002).

\section{Les objectifs des alliances}

11 On peut répartir les objectifs des compagnies rejoignant les alliances en trois types (Kleymann, 2002) :

- la défense de leur marché : l'entrée dans une alliance permet de défendre sa position sur son marché d'origine, en offrant l'opportunité à la compagnie d'y recentrer ses opérations et en laissant ses partenaires opérer les vols sur les autres marchés ;

- l'amélioration de l'efficacité des opérations en permettant aux compagnies de partager leurs infrastructures au sol, leurs services d'entretien des appareils et en harmonisant les horaires ;

- l'offensive vers de nouveaux marchés : en pouvant présenter leurs services sous une marque unique et plus prestigieuse, plus connue et à laquelle les gens font confiance, certaines compagnies peuvent améliorer leur image, ce qui facilite leurs ventes. Les alliances 
permettent également aux compagnies alliées d'utiliser les hubs de leurs partenaires dans chacune des régions comme base de leur trafic dans ces zones. Elles permettent aussi d'augmenter les parts de marchés sur les liaisons entre les hubs (Vowles, 2000). Ces liaisons sont celles qui génèrent le plus de clientèle « business », la plus intéressante financièrement (Lee, 2003).

12 Selon Merlin (2000), pour constituer une bonne alliance il faut réunir au moins une des plus grandes compagnies états-uniennes, une des principales compagnies européennes, au moins une grande compagnie asiatique, plus diverses autres compagnies. En 2005, les trois alliances regroupaient 36 compagnies, dont 24 des 50 plus grandes compagnies mondiales, majoritairement issues d'Europe (18), des États-Unis et du Canada (7) (voir tableau 1).

Tableau 1. Les compagnies membres des alliances en 2005.

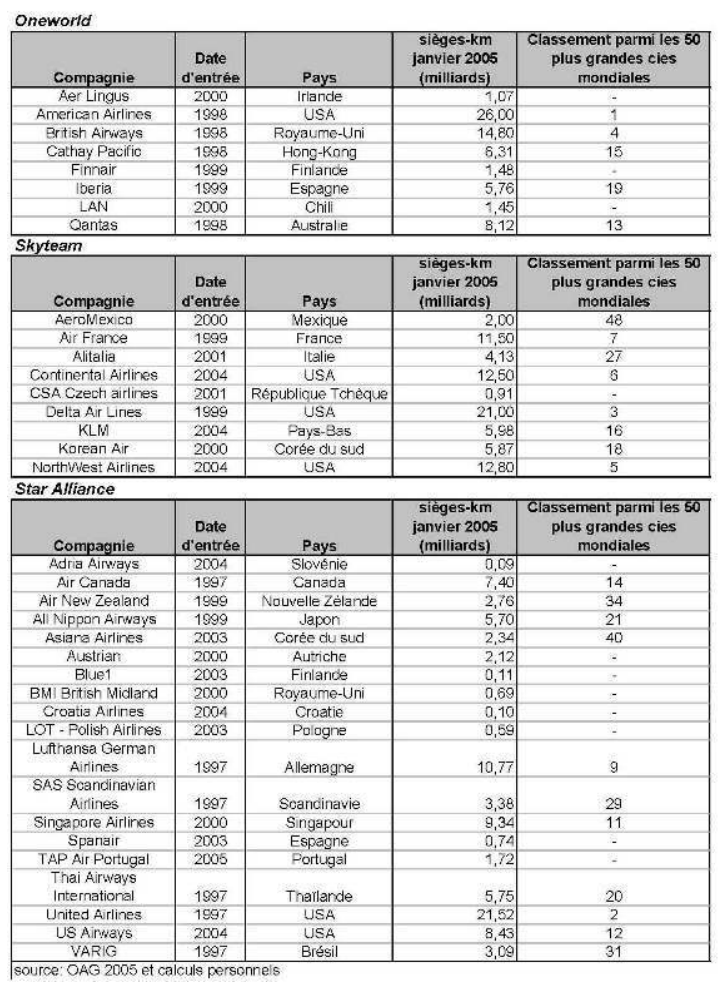

\section{Les compagnies low cost et les alliances}

Les compagnies low cost semblent pour l'instant exclues des alliances. Elles opèrent selon un réseau de point à point, sans hub ni possibilité de correspondance, en particulier en Europe et depuis des aéroports différents des compagnies «traditionnelles ». Cela rend moins intéressante et plus compliquée l'intégration de leur réseau (Kleymann, 2002).

Plus localement par contre, des alliances sont possibles, telle, par exemple, la coopération puis la fusion entre SN Brussels Airlines, compagnie traditionnelle, et Virgin Express, low cost, mais les deux compagnies opéraient depuis le même aéroport de base, Bruxelles National, ce qui a facilité la coordination de leur réseau.

15 Il est possible qu'à terme des alliances puissent se créer entre compagnies traditionnelles pour le trafic long-courrier et compagnies low cost pour les court-courriers, mais pour 
l'instant cela n'a pas été le cas. Au contraire, Aer Lingus et Varig, qui se sont mués partiellement en compagnies low cost depuis 2006, ont quitté leurs alliances respectives, Oneworld et Star Alliance ${ }^{1}$.

\section{L'organisation spatiale du trafic des alliances}

Pour comprendre la répartition spatiale des alliances de compagnies aériennes dans le monde, il faut poser le problème à différentes échelles : la répartition des alliances sera d'abord envisagée par pays et par région, puis selon les implantations par aéroports et enfin en fonction des villes mondiales. Cela permettra ensuite d'analyser les réseaux des alliances et de tenter de comprendre leur stratégie.

\section{La répartition du trafic des alliances par pays et par régions}

Concentrées dans les pays du centre, où leur part de trafic est supérieure à $40 \%$, les alliances commencent à s'étendre vers les pays de la périphérie les plus avancés. Certains pays émergents se distinguent par des taux plus élevés, tels le Mexique, la Thaïlande, le Chili, qui ont chacun une compagnie nationale membre d'une alliance (voir figure 1).

Figure 1. Part du trafic des alliances par pays en sièges en janvier 2005.

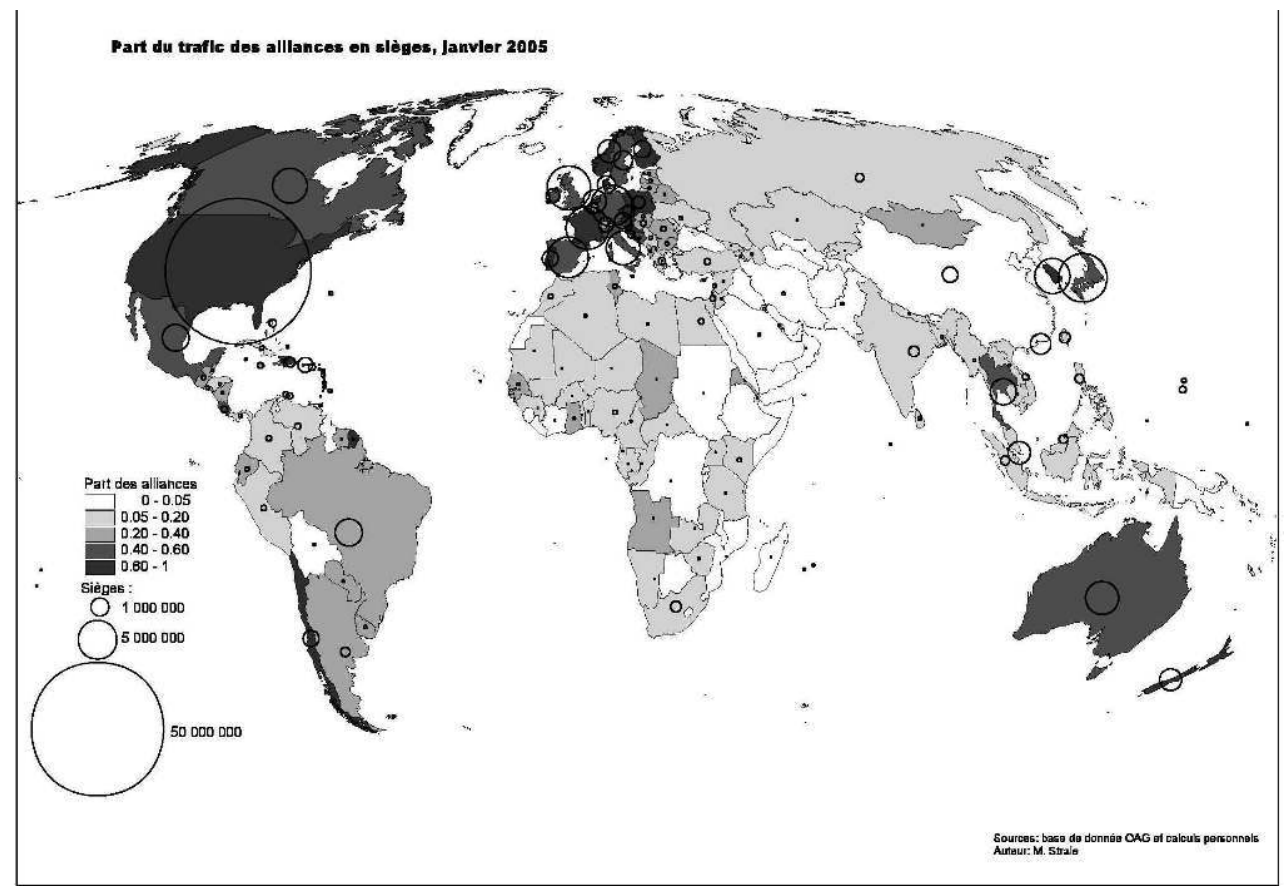

A l'inverse, on remarque les faibles taux de la Belgique, de la Suisse et de la Grèce au sein des pays du centre, liés à l'absence de compagnie membre en 2005.

Dans les régions mondiales les plus périphériques, l'Afrique et l'Asie centrale, aucune compagnie n'est membre d'une alliance ${ }^{2}$, ce qui est lié aux conditions d'accès dans les alliances : il faut répondre à des conditions financières, de qualité et de sécurité que n'atteignent pas toujours les compagnies d'Afrique et d'Asie. De plus, le faible trafic aérien à l'intérieur de ces régions rend peu intéressant l'installation d'un hub, et le trafic 
depuis le reste du monde peut être assuré depuis les grands hubs européens et d'Asie du Sud-Est.

Par ailleurs, aucune compagnie du Moyen-Orient n'est membre d'une des trois alliances mondiales de compagnies aériennes. Les raisons semblent ici être plutôt politiques. Plusieurs compagnies arabes projettent de créer leur propre alliance : l'Arab Air Carriers Organization (AACO), qui est à la base de ce projet, ne rassemblerait, au moins dans un premier temps, que des compagnies aériennes arabes.

La géographie mondiale du trafic des alliances à l'échelle des pays, avec de forts contrastes régionaux, révèle l'intérêt d'une analyse en fonction de régions homogènes. Le monde a été divisé en dix régions, qui serviront de base à l'analyse ; les trafics intra- ou inter-régionaux feront référence à ces délimitations de territoires (voir figure 2).

Figure 2. Part du trafic des alliances par et entre régions en sièges en janvier 2005.

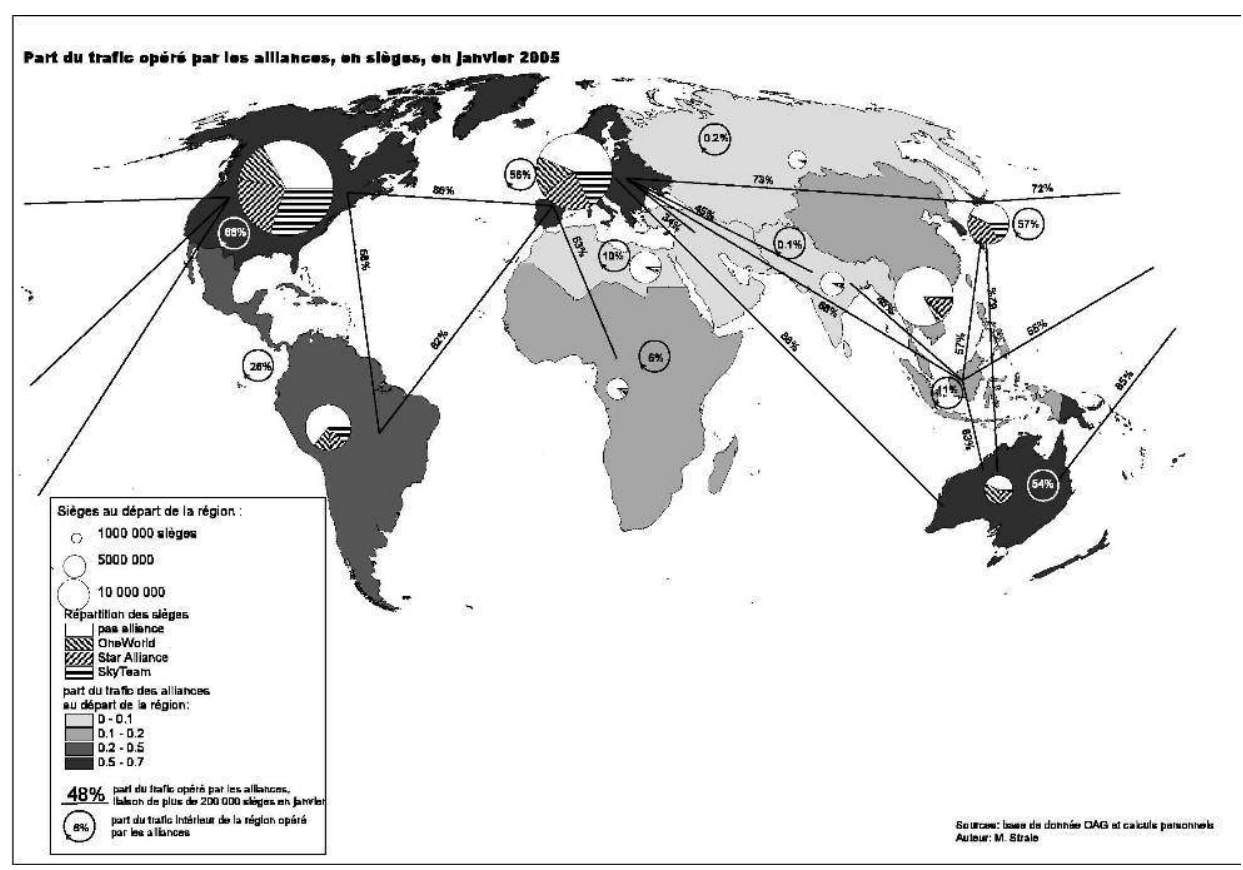

L'offre des alliances représente environ la moitié du total de l'offre aérienne régulière mondiale. Le tableau 2 , construit à partir des offres de sièges de janvier 2005 démontre la concentration de l'offre des alliances dans les régions centrales de l'économie; l'Amérique du nord, qui représente la moitié de l'offre totale des alliances, l'Europe et le Japon et la Corée du sud principalement. En revanche, elle est très faible dans les régions périphériques, ne dépassant pas les $15 \%$ en Afrique et en Asie centrale et de l'ouest. L'Asie du sud-est et l'Amérique latine sont en situation intermédiaire, reflet de leur intégration dans l'économie monde.

Tableau 2. Trafic aérien par région et part des alliances. 


\begin{tabular}{|c|r|r|r|}
\hline Région & $\begin{array}{c}\text { Trafic total au départ } \\
\text { de la région en } \\
\text { sièges (millions) }\end{array}$ & $\begin{array}{c}\text { Trafic total des } \\
\text { alliances, en sièges } \\
\text { (millions) }\end{array}$ & $\begin{array}{r}\text { Part des } \\
\text { alliances }\end{array}$ \\
\hline $\begin{array}{c}\text { Afrique du nord et } \\
\text { Moyen orient }\end{array}$ & 10,10 & 0,75 & $7,38 \%$ \\
\hline Afrique & 4,67 & 0,62 & $13,33 \%$ \\
\hline Amérique latine & 20,80 & 7,40 & $35,55 \%$ \\
\hline Amérique du nord & 91,35 & 61,15 & $66,94 \%$ \\
\hline Monde indien & 5,81 & 0,42 & $7,24 \%$ \\
\hline Asie de l'est et du sud- & & 6,10 & $17,19 \%$ \\
\hline est & 35,47 & 32,41 & $56,17 \%$ \\
\hline Europe & 57,70 & 9,78 & $57,35 \%$ \\
\hline Japon et Corée du sud & 17,06 & 4,54 & $55,77 \%$ \\
\hline Océanie et Pacifique & 8,13 & 0,22 & $6,70 \%$ \\
\hline Russie et Asie centrale & 3,34 & & \\
\hline Total & & $\mathbf{1 2 3 , 3 8}$ & $\mathbf{4 8 , 4 9 \%}$ \\
\hline
\end{tabular}

Source : OAG et calculs personnels

La part des alliances est toujours plus élevée pour les liaisons interrégionales que pour celles à l'intérieur des régions (figure 2) : elle les dépasse toujours de $35 \%$ et même de $60 \%$ pour les liaisons entre régions du centre. Les taux les plus élevés sont observés sur l'Atlantique nord ( $86 \%$ ) et la liaison entre l'Océanie et l'Amérique du nord ( $85 \%$ ). La part des alliances dans les vols intra-régionaux est plus faible et elle est même très faible pour l'Afrique, le monde arabo-musulman, le monde indien et la Russie et l'Asie centrale.

4 L'offre des alliances se concentre donc dans les régions riches et surtout sur les liaisons depuis et entre ces régions. Pour les vols à l'intérieur des régions ne comprenant pas de compagnie membre d'une des alliances, l'offre de ces dernières est faible. Les alliances y assurent surtout des vols long-courriers vers et depuis leur région d'origine.

5 Il serait intéressant de départager les vols nationaux et internationaux, notamment aux USA, et d'étudier la question du rôle des vols nationaux dans la stratégie des alliances, mais cela n'a pas été fait dans le cadre de cet article.

\section{Les implantations des alliances dans les grands aéroports}

Connaissant la géographie des alliances au niveau des pays et régions, passons à son analyse à plus grande échelle, celle des aéroports. Pour cela, leur trafic a été analysé au niveau des 500 plus grands aéroports mondiaux (voir figure 3). 
Figure 3. Part du trafic des alliances dans les 500 plus grands aéroports du monde, en janvier 2005.

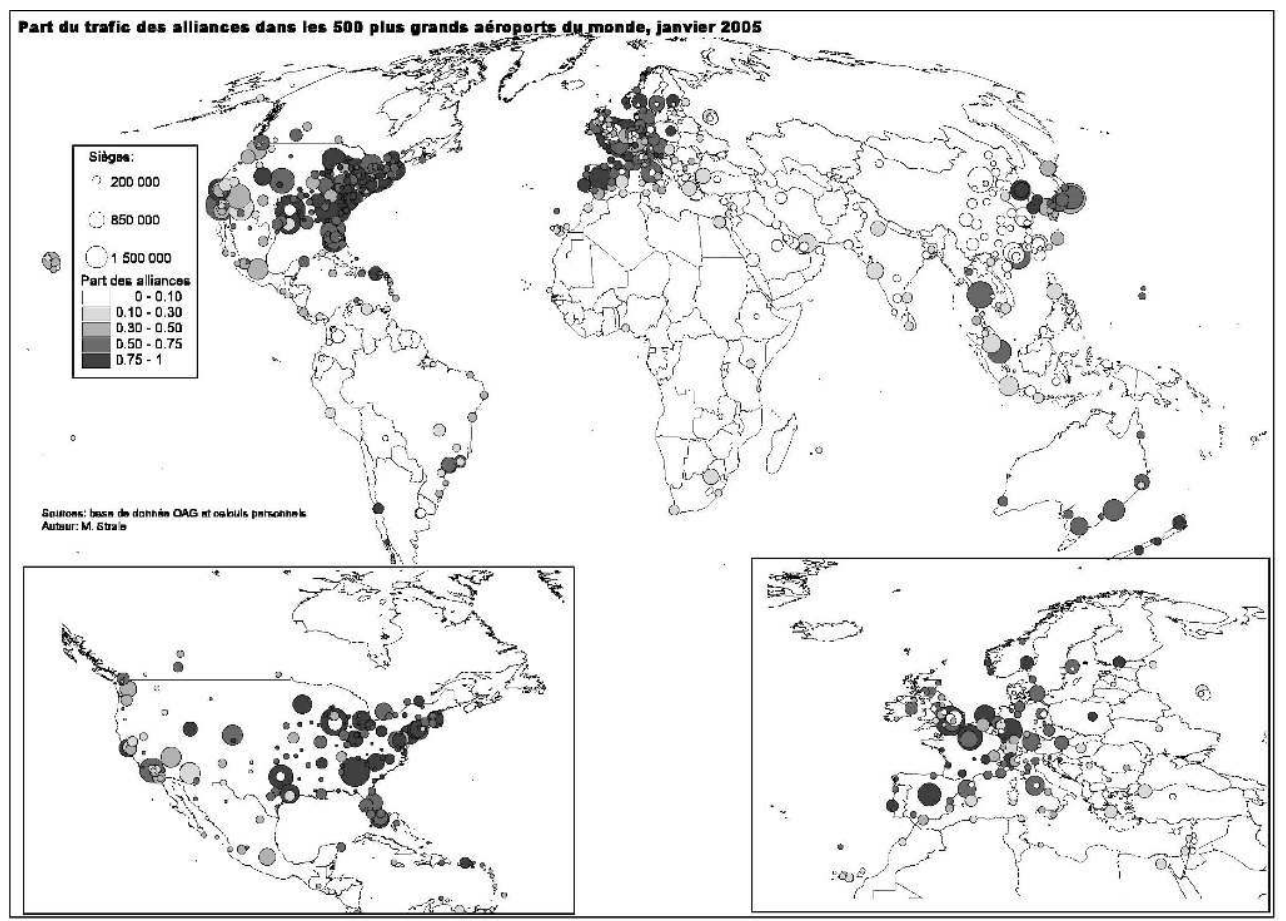

En Europe et en Amérique du nord, les plus grands aéroports ont des parts très élevées de trafic opéré par les alliances : ce sont les hubs des compagnies membres des alliances. On observe une offre relativement élévée des alliances en Afrique du nord et dans les Caraïbes, liée à l'offre touristiques des compagnies occidentales dans ces régions touristiques et proches.

L'offre des compagnies « low cost» se concentre dans de plus petits aéroports, où l'offre des alliances est très faible, voire nulle. C'est très visible pour les aéroports de Gérone, Rome Ciampino, Bergame et les seconds aéroports d'Oslo, Stockholm et Helsinki.

L'est et l'ouest des Etats-Unis sont nettement opposés, les taux les plus élevés étant observés à l'est, souvent supérieurs à $75 \%$, alors qu'à l'ouest, la part des alliances ne dépasse pas $50 \%$ dans de grands aéroports tels Phoenix, Las Vegas, Seattle. Cela est dû au fait que les deux plus grandes compagnies américaines non membres d'une alliance, Southwest (plus grande compagnie low cost américaine) et America West Airlines, organisent la majorité de leur vols dans l'ouest des États-Unis. Notons qu'America West Airlines a entre-temps fait faillite, puis a été reprise par US Airways et a été intégrée dans Star Alliance. L'offre importante des compagnies asiatiques, qui ne sont pas toutes membres des alliances, dans l'ouest des Etats-Unis explique aussi en partie le taux plus faible de trafic assuré par les alliances dans cette région.

En Asie du sud-est, trois grands aéroports, Hong-Kong, Bangkok et Singapour, se signalent par des taux élevés, car tous les trois sont des hubs de compagnies membres d'une des trois alliances. Leur localisation sur les flux de trafic entre l'Europe, l'Asie et l'Océanie, ainsi que leur intégration relativement bonne dans l'économie et l'existence d'un marché local expliquent cette situation. Hong-Kong et Singapour représentent une offre des alliances supérieure à celle de tout le reste de la région. Ces aéroports contrastent avec la très faible présence des alliances dans le reste de la région, surtout en Chine. 
31 A l'est des Philippines, les aéroports de Guam et de Tinian, situés dans les Mariannes du nord, possession des Etats-Unis, ressortent avec des parts de sièges opérés par les alliances supérieurs à $50 \%$, car ils servent de relais vers l'Océanie pour les compagnies aériennes américaines.

Si on considère les 500 plus grands aéroports mondiaux, on constate que peu d'entre eux sont situés hors des régions précitées, l'Afrique et l'Asie centrale étant particulièrement démunies.

En Amérique latine, la part des sièges des alliances dans les aéroports varie fort d'un pays à l'autre, selon qu'il y ait des membres d'alliance ou non. Quelques grands aéroports ressortent, Santiago, Sao Paulo et Rio de Janeiro ; ils sont les bases des compagnies Varig et LAN.

En Afrique, le constat est le même qu'au niveau national : l'offre des alliances est faible et le centre de l'Afrique est totalement dépourvu de grand aéroport.

Le Moyen-Orient a des parts de trafic des alliances très basses, seul l'aéroport de Dubaï, le plus grand de la région, et les aéroports de Beyrouth et de Tel-Aviv dépassant $10 \%$.

Afin de déterminer l'importance de la concentration de l'offre des alliances dans les plus grands aéroports, un coefficient de spécificité entre la taille des aéroports et la part des alliances dans le trafic au départ de ceux-ci a été construit. Les aéroports mondiaux ont été divisés en dix classes, des plus grands aux plus petits, reprenant chacune dix pourcents de l'offre au départ de tous les aéroports mondiaux. La part des alliances a été calculée dans chacune des classes et comparée à la moyenne.

Les alliances centrent leur offre sur les plus grands aéroports : elles sont sur-représentées dans les classes des plus grands aéroports, d'où elles organisent leur offre, notamment en formant des hubs de correspondance. Les petits aéroports sont moins intéressants ; ils peuvent être situés dans des régions marginales, être utilisés par les compagnies low cost, et les alliances y organisent surtout un rabattement du trafic vers leurs hubs principaux.

Figure 4. Coefficient de spécificité entre la taille des aéroports et la part des alliances.

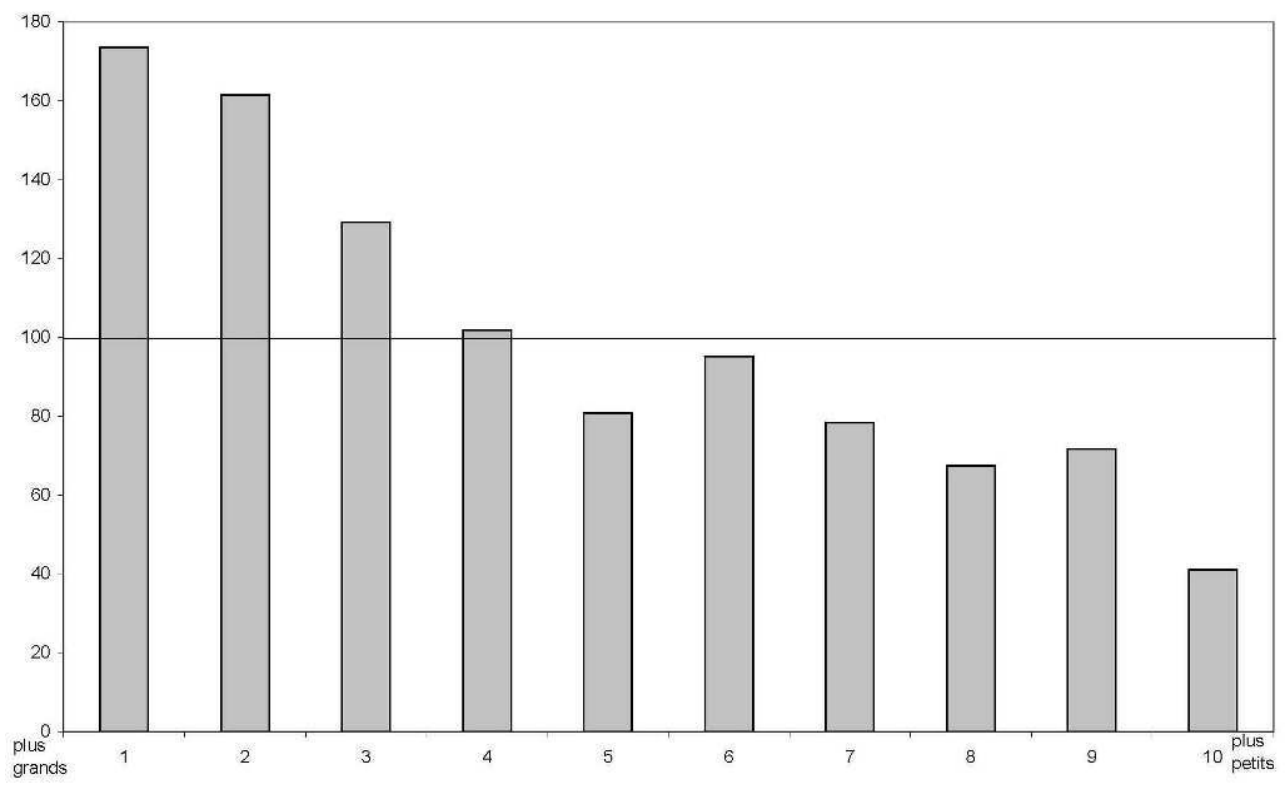

Source : OAG et calculs personnels 
Tableau 3. Part des alliances en sièges entre world cities.

\begin{tabular}{|c|r|r|r|r|}
\hline $\begin{array}{c}\text { World } \\
\text { Cities }\end{array}$ & \multicolumn{1}{|c|}{ Alpha } & \multicolumn{1}{|c|}{ Beta } & Gamma & Autre \\
\hline Alpha & 0,830 & \multicolumn{3}{|l|}{} \\
\hline Beta & 0,802 & 0,654 & & \\
\hline Gamma & 0,689 & 0,660 & 0,476 & \\
\hline Autre & 0,592 & 0,522 & 0,476 & \\
\hline
\end{tabular}

Source : OAG et calculs personnels

\section{La prééminence des villes mondiales}

L'empreinte de l'histoire et de la géopolitique pèse fortement sur la répartition des alliances, d'abord par la concentration dans les riches pays occidentaux, mais surtout à travers les lieux où sont présentes les alliances dans les régions périphériques. En Asie du sud-est et en Amérique latine, leur présence est en partie liée aux anciennes colonies occidentales (Lacoste Y., 2004). En général, la présence des alliances est plus forte en Amérique latine que dans les autres régions périphériques de l'économie monde, du fait, entre autre, de la présence des compagnies nord-américaines, reflet de la dépendance de l'Amérique latine par rapport à l'Amérique du nord. Les autres régions périphériques, Afrique, Asie centrale, Moyen-Orient se distinguent par une quasi absence des alliances, liée à des contraintes techniques à l'entrée des alliances, à des choix politiques, ou au peu d'intérêt économique de ces régions.

41 Les alliances globales de compagnies aériennes sont un exemple de la mondialisation de l'économie, influencée par les contextes politiques et économiques, et qui concerne surtout les pays riches, et quelques pays émergents d'Asie et d'Amérique latine, le reste du monde demeurant à l'écart. 


\section{La stratégie des alliances}

Les résultats observés sont-ils le fruit d'une stratégie des alliances ou, au contraire, leur développement se limite-t-il à additionner des compagnies pour couvrir le monde ? Pour y répondre, nous avons comparé les offres de 1995 et 2000 à la situation de 2005. L'évolution générale du trafic, l'évolution des liaisons hors des pays d'origine des compagnies ainsi que la question des partages de code seront envisagés.

\section{L'évolution du trafic des alliances : un recentrage de l'offre}

De 1995 à 2000, l'offre totale des compagnies membres des alliances a augmenté, puis s'est stabilisée. Cette stabilisation est due à la baisse du nombre de sièges et de vols à l'intérieur de l'Amérique du nord suite à la crise qui suivit le 11 septembre 2001. Si on ne tient pas compte des sièges sur les vols intra nord-américains, les sièges opérés par les compagnies membres des alliances en 2005 ont augmenté de plus de $40 \%$ entre 1995 et 2005.

Deux types de trafic se détachent sur le graphique: d'une part, les sièges sur les vols interrégionaux, les liaisons de plus de 200000 sièges et le trafic total sans les vols nordaméricains, qui ont le plus augmenté, environ $50 \%$ en dix ans ; d'autre part, les sièges sur les vols intra-régionaux, le trafic total et les petites liaisons de moins de 200000 sièges, qui ont connu une augmentation beaucoup plus faible, de l'ordre de 10 à $20 \%$.

Les compagnies « classiques ", membres des alliances, ont surtout augmenté leur offre sur les vols intercontinentaux et sur les plus importantes liaisons. A l'opposé, les vols à l'intérieur des régions, où la concurrence des low cost, notamment, est forte, et qui ont été les plus touchés par la crise du transport aérien aux États-Unis qui a suivi le 11 septembre, ont faiblement augmenté. Ils ont même connu une baisse depuis 2000. Les plus petites liaisons, souvent vers ou entre des régions périphériques, ont connu une faible croissance, reflet de leur faible développement et intérêt économique.

Cette évolution est la traduction du recentrage de l'offre des compagnies aériennes: Celles-ci ont concentré leur offre sur les plus grandes liaisons et sur les vols interrégionaux, plus rentables. Les sièges ont moins augmenté sur les plus petites liaisons, souvent plus éloignées des régions centrales d'où sont originaires les compagnies membres d'alliances. 
Figure 5. Évolution du trafic des compagnies membres des alliances en janvier 2005, en sièges $(1995=1)$.

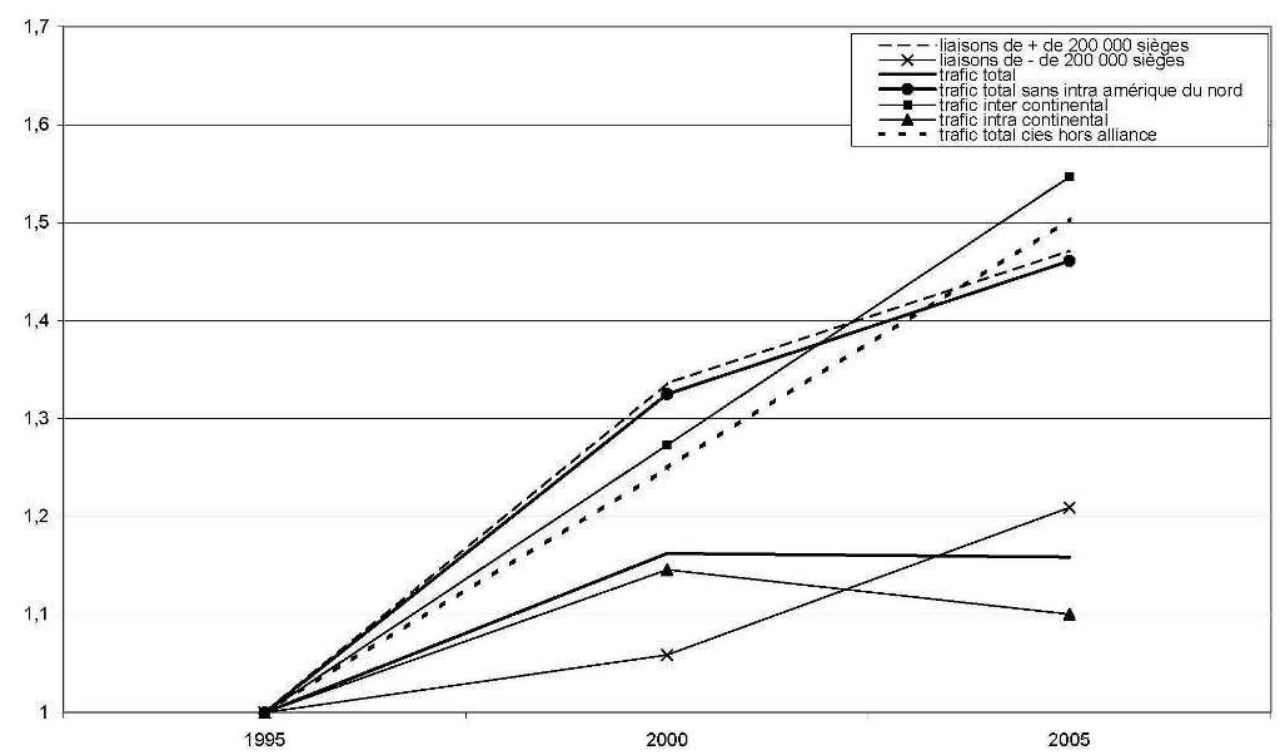

Source: OAG et calculs personnels

\section{Un délaissement des zones éloignées des régions d'origine des compagnies}

D’après plusieurs auteurs (Dennis, 2005 ; Kleymann, 2000 ; Hanlon, 1999), les compagnies tendent à recentrer leurs vols sur des liaisons partant de leur région ou pays d'origine pour limiter les coûts, laissant à leurs partenaires mieux situés le soin d'opérer les vols plus éloignés. Pour vérifier cette hypothèse, la part des sièges des compagnies n'ayant ni pour départ ni pour arrivée la région et le pays d'origine de la compagnie a été est calculée (voir tableau 4).

Tableau 4. Sièges offerts hors des pays et régions d'origine des compagnies membres des alliances.

\begin{tabular}{|l|r|r|r|}
\hline \multicolumn{1}{|c|}{ Sièges (en milliards) } & \multicolumn{1}{|c|}{1995} & \multicolumn{1}{c|}{$\mathbf{2 0 0 0}$} & \multicolumn{1}{c|}{$\mathbf{2 0 0 5}$} \\
\hline Totaux & 106,48 & 123,77 & 123,38 \\
\hline $\begin{array}{l}\text { hors de la région d'origine des } \\
\text { compagnies }\end{array}$ & 2,98 & 2,36 & 1,85 \\
\hline $\begin{array}{l}\text { part des sièges hors de la région } \\
\text { d'origine des compagnies }\end{array}$ & $2,8 \%$ & $1,9 \%$ & $1,5 \%$ \\
\hline hors du pays d'origine des compagnies & 4,08 & 3,34 & 2,97 \\
\hline $\begin{array}{l}\text { part des sièges hors du pays d'origine } \\
\text { des compagnies }\end{array}$ & $3,8 \%$ & $2,7 \%$ & $2,4 \%$ \\
\hline
\end{tabular}

\section{Source : OAG et calculs personnels}

Les sièges sur les vols dont ni le départ ni l'arrivée ne sont situés dans la région ou le pays d'origine de la compagnie, qui étaient déjà peu nombreux en 1995, avant la formation des alliances, ont fortement diminué en dix ans. Les alliances encouragent ce phénomène en permettant aux compagnies d'augmenter virtuellement le nombre de destinations 
qu'elles peuvent proposer à leurs clients grâce à la mise en commun de leur offre. Les compagnies n'ont pas besoin d'opérer des vols éloignés de leur région d'origine pour proposer des destinations dans le monde entier. On peut vérifier cette hypothèse en analysant les partages de codes entre les membres des alliances.

L'utilisation d'avions long-courriers de moyenne capacité, qui permettent d'aller plus loin sans imposer de dessertes intermédiaires pour contribuer au remplissage de l'avion, comme c'est le cas avec les avions de plus grande capacité, a sans doute aussi un impact sur la réduction des vols hors pays d'origine. Diverses compagnies ont remplacé des vols $\mathrm{X}-\mathrm{Y}-\mathrm{Z}$ par des vols X-Y et X-Z, ce qui fait diminuer ou disparaitre les vols extra-nationaux $Y-Z$. Mais cette question n'a pas été étudiée ici.

\section{Les partages de codes, une stratégie des alliances}

50 L'évolution des partages de codes est liée à la stratégie des alliances (voir tableau 5) : plus de la moitié des vols de Star Alliance et de Skyteam ont plusieurs codes appartenant à leurs partenaires. Oneworld, qui est considérée par plusieurs auteurs (Kleymann, 2002) comme l'alliance la moins intégrée, a une part de vols ayant des partages de codes beaucoup plus faible, mais en croissance également.

Tableau 5. Les partages de codes.

Part des vols ayant un partage de code avec:

\begin{tabular}{|c|c|c|c|c|c|c|}
\hline \multicolumn{2}{|c|}{ des partenaires de l'alliance } & \multicolumn{2}{c|}{$\begin{array}{c}\text { une compagnie membre d'une } \\
\text { alliance concurrente }\end{array}$} & $\begin{array}{c}\text { une compagnie non membre } \\
\text { d'une des trois alliances }\end{array}$ \\
\hline alliance & $\mathbf{2 0 0 0}$ & $\mathbf{2 0 0 5}$ & $\mathbf{2 0 0 0}$ & $\mathbf{2 0 0 5}$ & $\mathbf{2 0 0 0}$ & $\mathbf{2 0 0 5}$ \\
\hline Oneworld & $5,9 \%$ & $20,9 \%$ & $2,9 \%$ & $2,6 \%$ & $10,7 \%$ & $18,4 \%$ \\
\hline Star alliance & $22,8 \%$ & $54,8 \%$ & $1,6 \%$ & $0,3 \%$ & $10,6 \%$ & $12,0 \%$ \\
\hline Skyteam & $43,5 \%$ & $58,6 \%$ & $2,6 \%$ & $0,3 \%$ & $21,9 \%$ & $15,4 \%$ \\
\hline
\end{tabular}

Source : OAG et calculs personnels

51 La formation des alliances a entraîné une baisse des vols comportant des partages de codes entre compagnies membres d'alliances rivales. Ces accords commerciaux ne représentent plus que quelques pourcents des vols des alliances. Par contre, les accords avec des compagnies extérieures aux alliances tendent à se maintenir voire à se développer, témoins d'une seconde sphère de compagnies gravitant autour des alliances sans en être membres pour l'instant.

En absolu, ces accords ont plus que doublé entre 2000 et 2005, les hausses les plus fortes concernent les vols intérieurs nord-américains et pour les vols hors de la région d'origine de la compagnie (voir tableau 6). Pour les vols en Amérique du nord, cette hausse est due au fait que le marché est inaccessible pour les compagnies étrangères, la seule façon d'y proposer des sièges étant dès lors le partage de code. Les partages de codes sont aussi très nombreux entre compagnies américaines, le marché très concurrentiel et très vaste les empêchant de couvrir seules l'offre nord-américaine (Vowles, 2000). La hausse des partages de codes au sein des alliances sur les vols dont ni l'arrivée ni le départ ne sont dans la région d'origine des compagnies confirme un des rôles des alliances: elles permettent aux compagnies de recentrer leur offre sur leur région d'origine et, grâce aux partages de codes, de proposer des sièges loin de cette région et ainsi d'en tirer des bénéfices, sans devoir opérer le vol réellement. 


\begin{tabular}{|l|c|c|}
\hline Partages de codes & $\mathbf{2 0 0 0}$ & $\mathbf{2 0 0 5}$ \\
\hline Total (milliers) & 367,6 & 813,6 \\
\hline $\begin{array}{l}\text { hors région d'origine } \\
\text { de la cie (milliers) }\end{array}$ & 146,2 & 286,9 \\
\hline $\begin{array}{l}\text { vols intérieurs nord- } \\
\text { américains (milliers) }\end{array}$ & 240,8 & 544,1 \\
\hline
\end{tabular}

Source : OAG et calculs personnels

\section{Le rôle des compagnies aériennes dans les alliances}

British Airways domine les liaisons depuis l'Europe avec Iberia comme partenaire vers le sud et Finnair vers l'est. American Airlines contrôle sa région d'origine ainsi que l'importante liaison Amérique du nord - Amérique latine, plus de deux millions de sièges en janvier 2005. Qantas domine l'Océanie et les liaisons qui la relient au reste des régions. Cathay contrôle l'Asie du sud-est sauf pour les liaisons vers l'Europe et l'Océanie. LAN, compagnie chilienne, est limité à un rôle de partenaire régional pour l'Amérique latine. 
Figure 6. Carte des réseaux des compagnies des alliances.

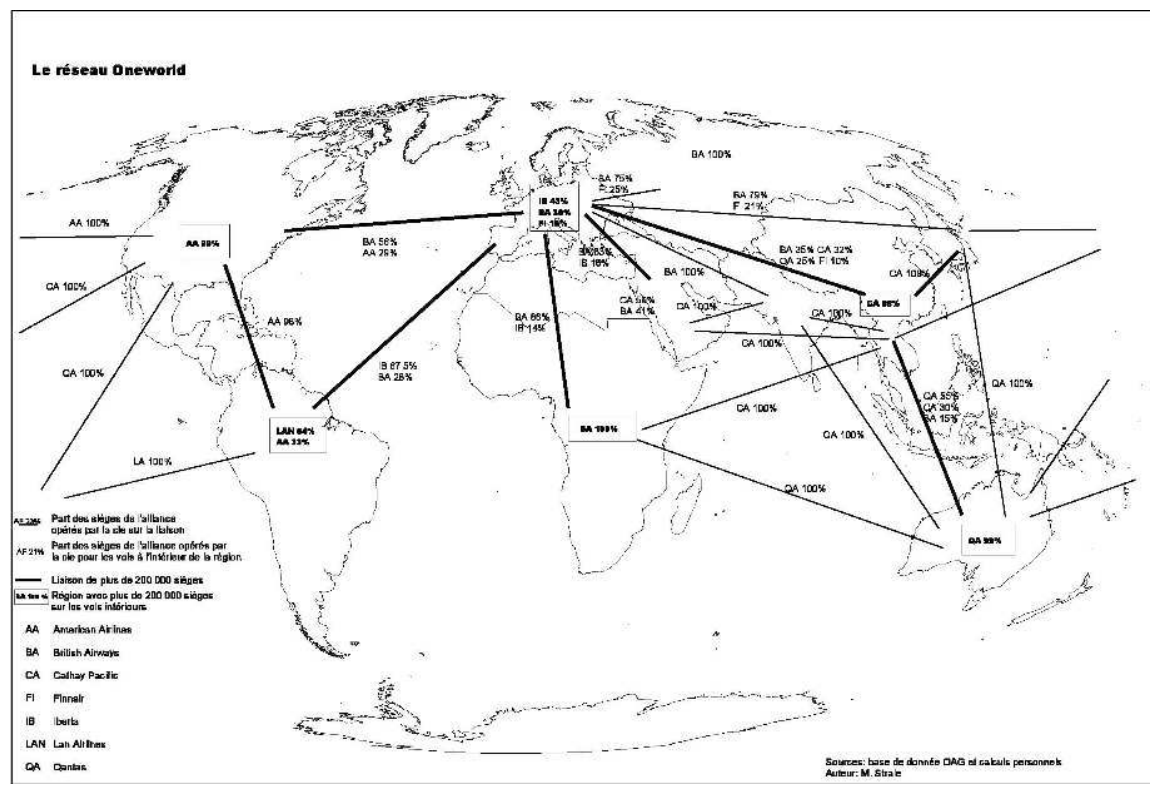

\section{Star Alliance (figure 7)}

Lufthansa domine les sièges en Europe et est présente sur toutes les liaisons depuis cette région, avec TAP comme partenaire vers le sud et Austrian vers l'est. En Amérique du nord, United domine les liaisons de plus de 200000 sièges sauf vers l'Amérique latine, domaine de US Airways. Air Canada est présent sur la plupart des liaisons depuis sa région d'origine. En Asie du sud-est, Thaï et Singapore dominent à deux les liaisons de la région. C'est le même système pour All Nippon Airways et Asiana au Japon et en Corée du sud. Air New Zealand et VARIG dominent seuls leur région d'origine et sont présents sur quelques liaisons. Sur l'Atlantique nord, on retrouve les quatre plus grandes compagnies de Star Alliance. 
Figure 7. Carte des réseaux des compagnies des alliances.

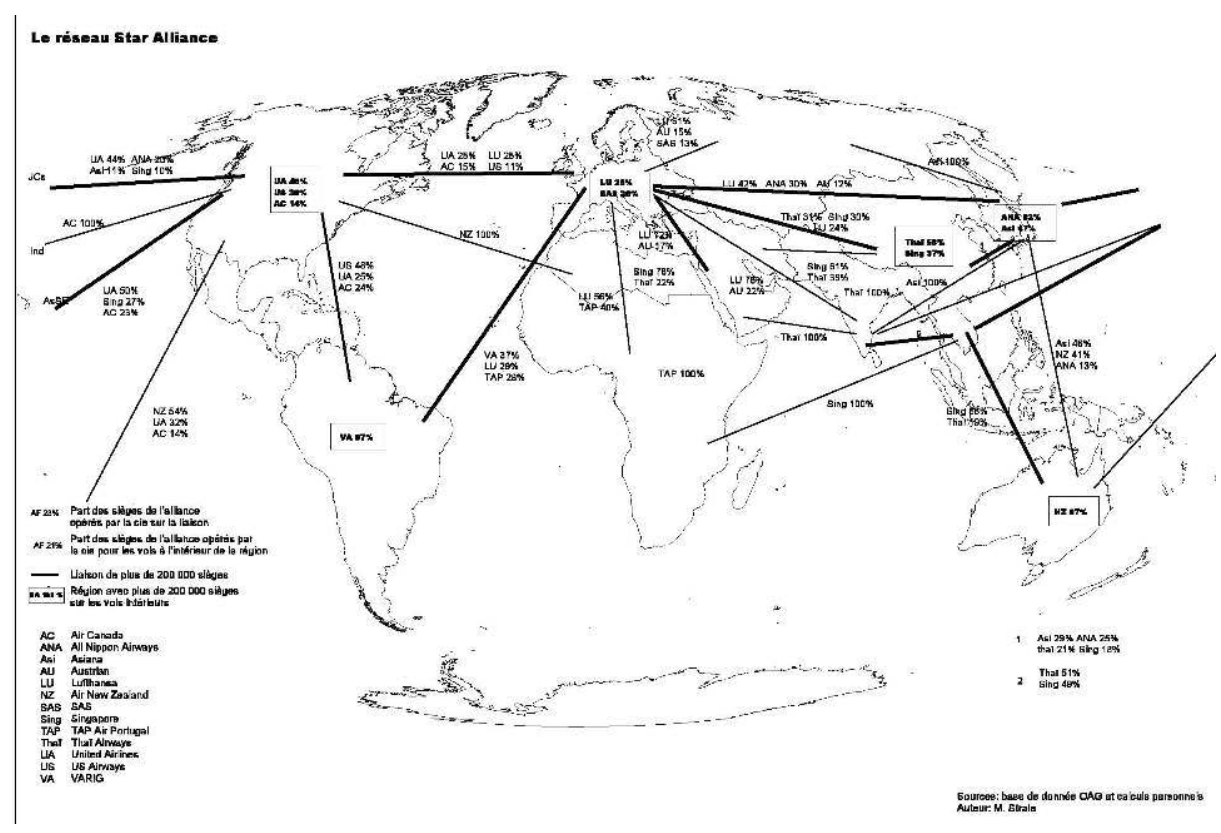

\section{Skyteam (figure 8)}

59 Air France et KLM contrôlent les liaisons depuis l'Europe, avec pour certaines d'entre elles la participation d'Alitalia ou de Czech Airlines. Les trois compagnies nordaméricaines de Skyteam se partagent le trafic depuis leur région, Continental Airlines vers l'Océanie et l'Amérique latine, Northwest surtout vers l'Asie, Delta vers l'Europe et l'Amérique latine. Korean Air, seule compagnie asiatique de l'alliance, domine sa région d'origine. Et enfin Aeromexico domine l'Amérique du sud et est présent sur les vols vers le nord du continent.

Comme dans le cas de Oneworld, les plus petites liaisons sont souvent entièrement dans les mains d'une ou de deux compagnies de Skyteam.

$61 \mathrm{Au}$ vu de ces trois cartes, des tendances communes apparaissent :

- l'Europe et l'Amérique du nord sont souvent dominées par une grande compagnie en partenariat avec d'autres en fonction des destinations ;

- le partenaire sud-américain est en général limité aux vols à l'intérieur de l'Amérique latine ;

- l'Atlantique nord est couvert par toutes les plus grandes des compagnies des alliances ;

- les sièges et les liaisons vers les régions périphériques sont limités. 
Figure 8. Carte des réseaux des compagnies des alliances.

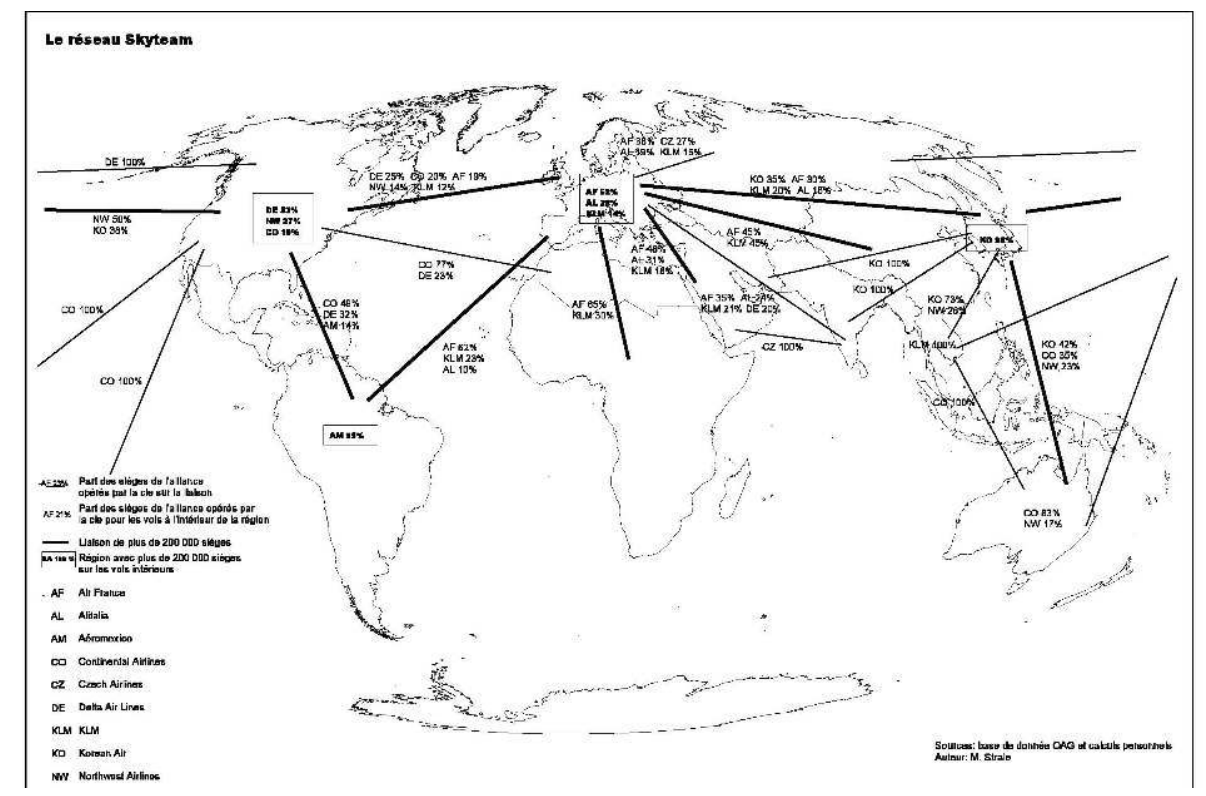

\section{La spécialisation des compagnies aériennes}

Afin de mieux cerner la spécificité des compagnies membres des alliances, nous avons réalisé une analyse en composantes principales reprenant pour chaque compagnie membre d'une des trois alliances, leur taille, leur importance dans l'alliance, ainsi que le type de trafic et les régions où elles opèrent. Grâce aux résultats et aux cartes précédentes des réseaux des compagnies, on peut diviser les compagnies en une dizaine de classes (voir tableau 7). 
Tableau 7. Tableau du classement des compagnies.

\begin{tabular}{|c|c|c|c|c|}
\hline compagnie & alliance & \begin{tabular}{|l|} 
sièges-km \\
realisés (SKR) en
\end{tabular} & $\begin{array}{c}\text { part des SKR de } \\
\text { lalliance }\end{array}$ & $\begin{array}{l}\text { part des vols dans la } \\
\text { région d'orignie }\end{array}$ \\
\hline American Airlines & Oneworld & 26,0 & 0,400 & 0.759 \\
\hline Delta Air Lines & Skyteam & 21,0 & 0,274 & 0,930 \\
\hline United Airlines & Star Alliance & 21,5 & 0,248 & 0,882 \\
\hline Grandes compagnies americain & & 22,8 & 0,307 & 0.857 \\
\hline Air France & Skyteam & 11,5 & 0,150 & 0,754 \\
\hline British Airways & Oneworld & 14,8 & 0.228 & 0.604 \\
\hline Lutthansa German Airlines & Star Alliance & 10,8 & 0,124 & 0,751 \\
\hline Grandes compagnies européen & & 12.4 & 0,167 & \\
\hline Air Canada & Star Alliance & 7,4 & 0,085 & 0,820 \\
\hline Continental Airlines & Skyteam & 125 & 0163 & 0770 \\
\hline Northwest Airlines & Skyteam & 12,8 & 0,167 & 0,890 \\
\hline US Airways & Star Alliance & 8.4 & 0,097 & 0.924 \\
\hline Compagnies américaines de tail & ile moyenne & 10,3 & 0,128 & 0,851 \\
\hline Alitalia & Skyteam & 4,1 & 0,054 & 0,833 \\
\hline Austrian & Star Alliance & 2.1 & 0.024 & 0.736 \\
\hline Czech Airlines & Skyteam & 0.9 & 0.012 & 0,823 \\
\hline Finnair & Oneworld & 1,5 & 0,023 & 0,872 \\
\hline iberia & Oneworld & 5,6 & 0,089 & 0,888 \\
\hline KLM-Royal Dutch Airlines & Skyteam & 6,0 & 0,078 & 0,610 \\
\hline SAS Scandinavian Airlines & Star Alliance & 3,4 & 0,039 & 0,937 \\
\hline TAP Air Portugal & Star Alliance & 1,7 & 0,020 & 0.835 \\
\hline Compagnies européennes de ta & aille moyenne & 3,2 & 0,042 & 0,817 \\
\hline All Nippon Airways & Star Alliance & 5,7 & 0.066 & 0,909 \\
\hline Asiana Airlines & Star Alliance & 2,3 & 0,027 & 0,659 \\
\hline Cathay Pacific & Oneworld & 6,3 & 0,097 & 0,482 \\
\hline Korean Air & Skyteam & 5,9 & 0.077 & 0,645 \\
\hline Singapore Airlines & Star Alliance & 9,3 & 0,108 & 0.449 \\
\hline Thai Airways International & Star Alliance & 58 & 0.066 & 0,035 \\
\hline Grandes compagnies asiatiques & & 5,9 & 0.073 & 0,630 \\
\hline Aeromexico & Skyteam & 2,0 & 0.026 & 0,816 \\
\hline Lan Airlines & Oneworld & 1,5 & 0,022 & 0,870 \\
\hline VARIG & Star Alliance & 3.1 & 0.036 & 0,914 \\
\hline Compagnies d'Amérique latine & & $\frac{1,2}{2,2}$ & 0,028 & 0,867 \\
\hline Air New Zealand & Star Alliance & 2,8 & 0,032 & 0,848 \\
\hline Qantas & Onewtorld & 8.1 & 0.125 & 0,803 \\
\hline Compagnies d'Océanie & & 5,4 & 0.078 & 0,825 \\
\hline Adria Airways & Star Alliance & 0,1 & 0.001 & 0.849 \\
\hline Aer Linqus & Onewarta & 11 & 0.016 & $0852>2$ \\
\hline Blue1 & Star Alliance & 0,1 & 0,001 & 1,000 \\
\hline Bmi british midland & Star Alliance & 0.7 & 0.008 & 0.953 \\
\hline Croatia Airlines & Star Alliance & 0,1 & 0,001 & 1,000 \\
\hline LOT - Polish Airlines & Star Alliance & 0,6 & 0.007 & 0.876 \\
\hline Spanair & Star Alliance & 0.7 & 0,009 & 0,997 \\
\hline Petites compagnies européenne & & 0.5 & 0.006 & 0.932 \\
\hline
\end{tabular}

\section{Les grandes compagnies américaines}

Ces compagnies proposent une offre mondiale, dominent les sièges de leur alliance dans leur région d'origine et opèrent sur la majorité des liaisons depuis l'Amérique du nord. Elles représentent au moins $25 \%$ des sièges de leur alliance (voir tableau 7). Elles ont une offre importante vers l'Europe et l'Amérique latine, principales liaisons depuis l'Amérique du nord.

En comparant la situation actuelle à celle de 1995 et 2000, au tableau 8, on observe que l'importance de ces compagnies pour les vols reliant l'Amérique du Nord au reste du monde s'est accrue, même si elles opéraient déjà de nombreux vols vers l'extérieur en 1995, avant de former des alliances. Le trafic international correspond aux vols sortant ou entrant en Amérique du nord. Dans un contexte de crise depuis 2001, qui a entraîné la baisse du trafic total de ces compagnies, cette croissance des sièges internationaux est remarquable, et entre 2000 et 2005 elle est plus rapide que celle de la moyenne des alliances.

Tableau 8. Évolution du trafic des grandes compagnies américaines.

\begin{tabular}{|l|r|r|r|}
\cline { 2 - 4 } \multicolumn{1}{c|}{} & \multicolumn{1}{|c|}{1995} & \multicolumn{1}{c|}{$\mathbf{2 0 0 0}$} & \multicolumn{1}{c|}{2005} \\
\hline trafic total (millions) & 39,71 & 41,85 & 39,09 \\
\hline trafic international (millions) & 4,19 & 4,21 & 5,00 \\
\hline part du trafic international & $10,6 \%$ & $10,1 \%$ & $12,8 \%$ \\
\hline
\end{tabular}

Source : OAG et calculs personnels 


\section{Les grandes compagnies européennes}

65 d'origine et la majorité des liaisons depuis l'Europe avec différents partenaires selon les liaisons. La part des sièges de chacune de ces compagnies sur les liaisons sortant de l'Europe est d'au moins $25 \%$ (voir tableau 7).

Ces compagnies, qui opéraient déjà de nombreux vols vers le reste du monde, ont renforcé ces liaisons depuis 1995 (voir tableau 9). Malgré un léger repli de leur trafic total en sièges, entre 2000 et 2005, le trafic international de ces trois grandes compagnies, déjà membres d'alliances en 2000, a continué à augmenter. Elles ont renforcé leur rôle de grande compagnie internationale européenne au sein des alliances.

Tableau 9. Évolution du trafic des grandes compagnies européennes.

\begin{tabular}{|l|r|r|r|}
\cline { 2 - 4 } \multicolumn{1}{c|}{} & \multicolumn{1}{c|}{1995} & \multicolumn{1}{c|}{$\mathbf{1 9 0 0 0}$} & \multicolumn{1}{c|}{$\mathbf{2 0 0 5}$} \\
\hline trafic total (millions) & 9,82 & 16,36 & 16,15 \\
\hline trafic international (millions) & 2,35 & 3,75 & 4,32 \\
\hline part du trafic international & $23,9 \%$ & $22,9 \%$ & $26,8 \%$ \\
\hline
\end{tabular}

Source : OAG et calculs personnels

\section{Les compagnies américaines de taille moyenne}

Les compagnies de ce groupe représentent environ $15 \%$ de l'offre de l'alliance dont elles sont membres (voir tableau 7). Leur offre sur les vols long-courriers ne couvre pas toutes les liaisons depuis l'Amérique du nord mais elles sont toutes présentes sur l'Atlantique nord. Elles ont souvent spécialisé leur offre internationale sur quelques régions.

En comparant l'évolution du total des sièges à celle des sièges sur les liaisons interrégionales, on observe que l'offre a le plus augmenté sur les liaisons internationales où les compagnies représentent plus de $10 \%$ des sièges de leur alliance (voir tableau 10). Par ailleurs, on constate que ces compagnies ont plus souffert de la crise que les grandes, le nombre total de sièges ayant régressé entre 1995 et 2005. 
Tableau 10. Évolution du trafic des compagnies américaines de taille moyenne.

\begin{tabular}{|l|r|r|r|}
\cline { 2 - 4 } \multicolumn{1}{c|}{} & \multicolumn{1}{c|}{1995} & \multicolumn{1}{c|}{$\mathbf{2 0 0 0}$} & \multicolumn{1}{c|}{$\mathbf{2 0 0 5}$} \\
\hline trafic total (millions) & 27,45 & 27,75 & 25,44 \\
\hline $\begin{array}{l}\text { trafic international, offre } \\
\text { de + de 10\% sur la } \\
\text { liaison (millions) }\end{array}$ & 1,70 & 2,44 & 3,28 \\
\hline $\begin{array}{l}\text { trafic international total } \\
\text { (millions) }\end{array}$ & 2,09 & 2,72 & 3,53 \\
\hline $\begin{array}{l}\text { part du trafic } \\
\text { international, offre de }+ \\
\text { de 10\% sur la liaison }\end{array}$ & $6 \%$ & & $13 \%$ \\
\hline part du trafic international & $8 \%$ & $10 \%$ & $14 \%$ \\
\hline
\end{tabular}

Source: OAG et calculs personnels

69 Ces résultats confirment que ce qui avait été observé précédemment, à savoir la participation des compagnies moyennes à certaines liaisons depuis l'Amérique et pas à d'autres, ce qui est en partie le résultat d'une stratégie: les compagnies ont proportionnellement plus augmenté les sièges sur ces liaisons que sur les autres. Leur profil dans les alliances est partiellement lié à l'évolution récente, depuis que ces compagnies en sont membres.

\section{Les compagnies européennes de taille moyenne}

70 Les membres de ce groupe sont souvent les deuxièmes ou troisièmes compagnies européennes en nombre de sièges dans l'alliance dont elles font partie (voir tableau 7). Elles opèrent sur une sélection de liaisons en partenariat avec les grandes compagnies européennes.

71 Ce groupe a connu une forte croissance de 1995 à 2005, plus de $50 \%$, croissance proche de celle des grandes compagnies européennes (voir tableau 11). Le nombre des sièges sur les destinations internationales a augmenté encore plus fortement, particulièrement ceux correspondant aux liaisons où les compagnies européennes de taille moyenne représentent plus de $10 \%$ de l'offre de leur alliance, qui ont presque doublé.

Comme pour les classes précédentes, ces compagnies ont renforcé leur rôle dans les alliances depuis leur entrée. 
Tableau 11. Évolution du trafic des compagnies européennes de taille moyenne.

\begin{tabular}{|l|r|r|r|}
\cline { 2 - 4 } \multicolumn{1}{c|}{} & \multicolumn{1}{c|}{1995} & \multicolumn{1}{c|}{$\mathbf{2 0 0 0}$} & \multicolumn{1}{c|}{$\mathbf{2 0 0 5}$} \\
\hline trafic total (millions) & 9,96 & 13,80 & 15,34 \\
\hline $\begin{array}{l}\text { trafic international, offre } \\
\text { de + de 10\% sur la } \\
\text { liaison (millions) }\end{array}$ & 0,90 & 1,37 & 1,77 \\
\hline $\begin{array}{l}\text { trafic international total } \\
\text { (millions) }\end{array}$ & 1,55 & 2,13 & 2,49 \\
\hline $\begin{array}{l}\text { part du trafic } \\
\text { international, offre de + } \\
\text { de 10\% sur la liaison }\end{array}$ & $9 \%$ & $10 \%$ & $12 \%$ \\
\hline part du trafic international & $16 \%$ & $15 \%$ & $16 \%$ \\
\hline
\end{tabular}

Source : OAG et calculs personnels

\section{Les compagnies asiatiques}

Ce groupe reprend tous les membres asiatiques des trois alliances. Entre 1995 et 2005, ces compagnies ont connu une forte croissance du trafic international sortant de leur région d'origine (voir tableau 12). Cela confirme leur rôle de compagnie internationale dominant leur région d'origine.

Dans ce contexte, il est intéressant de constater que les sièges que ces compagnies proposent vers l'Amérique du Nord ont diminué depuis 2000, alors que cette liaison connaissait une croissance. Les compagnies asiatiques ont augmenté les sièges et la part qu'ils représentaient dans le total de 1995 à 2000, ensuite leur offre a diminué de 2000 à 2005 , alors que les compagnies américaines membres des alliances ont connu l'évolution inverse. Entre 2000 et 2005, presque toutes ces compagnies étaient déjà membres des alliances et il semble que les compagnies asiatiques membres des alliances ont dû abandonner une partie des sièges sur le Pacifique nord au profit de leurs partenaires américains. Sur les liaisons avec l'Europe et avec l'Océanie on n'observe pas les mêmes résultats: l'évolution de l'offre des compagnies asiatiques est parallèle à l'évolution générale sur ces liaisons; elles n'y perdent pas de part de marché au profit de leurs alliées. 
Tableau 12. Évolution du trafic des compagnies asiatiques.

\begin{tabular}{|l|r|r|r|}
\cline { 2 - 4 } \multicolumn{1}{c|}{} & \multicolumn{1}{c|}{1995} & \multicolumn{1}{c|}{2000} & \multicolumn{1}{c|}{2005} \\
\hline trafic total (millions) & 11,70 & 13,45 & 15,48 \\
\hline trafic international (millions) & 2,74 & 3,66 & 4,79 \\
\hline part du trafic international & $23 \%$ & $27 \%$ & $31 \%$ \\
\hline
\end{tabular}

Trafic des compagnies membres des alliances en 2005 entre l'Amérique du

\begin{tabular}{|l|r|r|r|}
\cline { 2 - 4 } \multicolumn{1}{c|}{} & \multicolumn{1}{c|}{ nord et l'Asie } & \multicolumn{1}{c|}{$\mathbf{2 0 0 0}$} & $\mathbf{2 0 0 5}$ \\
\hline Total (millions) & 1995 & 1,46 & 1,55 \\
\hline compagnies asiatiques & 1,32 & & \\
(millions) & 0,42 & 0,59 & 0,56 \\
\hline part des cies asiatiques & $32 \%$ & $40 \%$ & $36 \%$ \\
\hline $\begin{array}{l}\text { compagnies américaines } \\
\text { (millions) }\end{array}$ & 0,84 & 0,79 & 0,92 \\
\hline part des cies américaines & $64 \%$ & $55 \%$ & $60 \%$ \\
\hline
\end{tabular}

Source : OAG et calculs personnels

\section{Les compagnies latino-américaines}

Trois compagnies font partie de ce groupe ; elles représentent moins de $5 \%$ de l'offre de leur alliance et leur trafic est très concentré sur les vols intérieurs à l'Amérique latine (voir tableau 7). Les liaisons lucratives vers l'Europe et l'Amérique du nord sont presque exclusivement aux mains des membres européens et nord-américains des alliances. Les compagnies de cette classe ont connu une hausse de leur trafic total en sièges de 1995 à 2005, tandis que les sièges vers les autres régions du monde ont crû jusque 2000 et diminué ensuite (voir tableau 13). Cette baisse est due à la diminution de l'offre de ces compagnies vers l'Amérique du nord après 2000 alors que cette liaison connaissait une très forte croissance.

Cette évolution est assez proche de celle observée pour les compagnies asiatiques : les compagnies sud-américaines membres des alliances ont diminué leur offre vers l'Amérique du nord au profit de leurs partenaires du nord du continent. 
Tableau 13. Évolution du trafic des compagnies latino-américaines.

\begin{tabular}{|l|r|r|r|}
\cline { 2 - 4 } \multicolumn{1}{c|}{} & \multicolumn{1}{c|}{$\mathbf{1 9 9 5}$} & \multicolumn{1}{c|}{$\mathbf{2 0 0 0}$} & \multicolumn{1}{c|}{$\mathbf{2 0 0 5}$} \\
\hline trafic total (millions) & 3,24 & 3,93 & 4,25 \\
\hline $\begin{array}{l}\text { trafic international } \\
\text { (millions) }\end{array}$ & 0,48 & 0,58 & 0,55 \\
\hline $\begin{array}{l}\text { part trafic } \\
\text { international }\end{array}$ & $15 \%$ & $15 \%$ & $13 \%$ \\
\hline
\end{tabular}

Trafic des compagnies membres des alliances en 2005 entre l'Amérique du nord et l'Amérique latine

\begin{tabular}{|l|c|r|r|}
\hline trafic total (millions) & 3,57 & 3,91 & 5,19 \\
\hline $\begin{array}{l}\text { cies sud-américaines } \\
\text { (millions) }\end{array}$ & 0,31 & 0,44 & 0,34 \\
\hline
\end{tabular}

Source : OAG et calculs personnels

\section{Les compagnies d'Océanie}

Deux compagnies font partie de ce groupe, elles dominent tous les deux l'Océanie au sein de leur alliance.

Leur trafic total a augmenté de plus de $50 \%$ en dix ans, mais, parallèlement, les sièges qu'elles proposaient vers le reste du monde ont diminué (voir tableau 14). Par contre, sur les liaisons où ces compagnies représentent plus de $10 \%$ de l'offre de leur alliance, les sièges ont augmenté, toutefois moins rapidement que le trafic total. C'est un signe de la concentration du réseau de ces compagnies sur leur région d'origine et sur les liaisons internationales principales.

Tableau 14. Évolution du trafic des compagnies d'Océanie.

\begin{tabular}{|l|r|r|r|}
\cline { 2 - 4 } \multicolumn{1}{c|}{} & \multicolumn{1}{c|}{1995} & \multicolumn{1}{c|}{$\mathbf{2 0 0 0}$} & \multicolumn{1}{c|}{$\mathbf{2 0 0 5}$} \\
\hline trafic total (millions) & 2,95 & 3,49 & 4,32 \\
\hline trafic international (millions) & 0,84 & 0,80 & 0,79 \\
\hline part du trafic international & $29 \%$ & $23 \%$ & $18 \%$ \\
\hline
\end{tabular}

Source : OAG et calculs personnels

Les petites compagnies européennes

Ces compagnies n'apparaissent nulle part sur les cartes des réseaux des compagnies des alliances, car leur taille est trop petite pour atteindre $10 \%$ de l'offre de leur alliance sur une liaison. Leur offre ne dépasse jamais $2 \%$ du total des sièges de l'alliance et est généralement inférieure au pourcent. Leurs destinations sont presque exclusivement européennes (voir tableau 7). 

En faisant la différence entre le trafic international court, vers le monde arabo-musulman et vers la Russie et l'Asie centrale, et le trafic international long vers les autres régions, on voit que c'est ce dernier qui a crû le plus rapidement, contrairement à ce qu'on aurait pu penser. Si cette évolution continue, ces compagnies vont peut-être adopter un profil plus proche des compagnies européennes de taille moyenne.

C'est le seul groupe dont l'évolution est difficilement explicable au vu du rôle des compagnies dans les alliances, ce qui est sans doute dû à leur entrée récente dans les alliances.

Tableau 15. Évolution du trafic des petites compagnies européennes.

\begin{tabular}{|l|r|r|r|}
\cline { 2 - 4 } \multicolumn{1}{c|}{} & \multicolumn{1}{c|}{$\mathbf{1 9 9 5}$} & \multicolumn{1}{c|}{$\mathbf{2 0 0 0}$} & \multicolumn{1}{c|}{$\mathbf{2 0 0 5}$} \\
\hline trafic total (millions) & 1,64 & 3,13 & 3,31 \\
\hline $\begin{array}{l}\text { trafic international court } \\
\text { (millions) }\end{array}$ & 0,03 & 0,02 & 0,04 \\
\hline $\begin{array}{l}\text { trafic international long } \\
\text { (millions) }\end{array}$ & 0,08 & 0,16 & 0,19 \\
\hline $\begin{array}{l}\text { trafic international } \\
\text { (millions) }\end{array}$ & 0,11 & 0,17 & 0,22 \\
\hline
\end{tabular}

Source: OAG et calculs personnels

Neuf groupes sur dix ont connu une évolution conforme à leur rôle dans les alliances. Les groupes de compagnies internationales ont augmenté leur offre internationale, les compagnies spécialisées sur certaines liaisons y ont augmenté leur offre... Aucun groupe n'a connu de transformation radicale, ce qui aurait été peu probable sur une durée de dix ans. Les alliances ont choisi leurs membres en fonction des marchés qu'elles devaient couvrir, et à la suite de leur intégration, ces membres ont évolué, se sont spécialisés pour correspondre au mieux au profil voulu dans leur alliance. Cela participe de la rationalisation et la spécialisation de l'offre des compagnies aériennes, stratégie encouragée par les alliances.

Une autre constatation intéressante est le gain de parts de marché sur les liaisons vers l'Asie et l'Amérique latine, au détriment de leurs partenaires dans ces régions. La formation des alliances semble avoir bénéficié aux compagnies des Etats-Unis et du Canada pour ces liaisons importantes. Cette évolution ne se retrouve pas sur les liaisons entre l'Europe et l'Asie ou l'Amérique latine, ni entre l'Amérique du nord et l'Océanie. C'est peut-être le résultat d'une stratégie au sein des alliances pour atténuer les effets de la crise sur le marché intérieur nord-américain, et qui se ferait au détriment des compagnies des pays émergeants, membres des alliances.

Un troisième constat se dégage avec évidence : les trois alliances ont au moins un membre dans presque chacune de ces classes. Lorsque ce n'est pas le cas, des stratégies sont mises en place pour pallier cette lacune, même si celle-ci se répercute malgré tout sur l'offre de l'alliance.

Ainsi Oneworld ne compte pas de membre dans la classe des compagnies américaines de taille moyenne, mais le fait d'avoir comme membre la plus grande compagnie mondiale, American Airlines, semble répondre en partie à ce manque. Néanmoins c'est l'alliance qui 
a l'offre la plus faible à l'intérieur de l'Amérique du nord. Cette alliance n'a pas non plus de membre au Japon ou en Corée du sud, mais ce manque a été résolu par l'entrée de Japan Air Lines dans l'alliance en $2006^{3}$. évidence leur stratégie spatiale, d'abord centrée sur l'Amérique du nord, l'Europe, le Japon et la Corée du sud, et l'Océanie. Un réseau moins dense, mais important pour la couverture mondiale, se développe en l'Amérique latine et en Asie du sud-est essentiellement. Des zones entières restent à l'écart de ce mouvement: l'Afrique, le Moyen-Orient et l'Asie centrale, situés en périphérie du réseau des alliances. L'intégration des régions dans les réseaux des alliances reflète leur insertion dans l'économie monde.

91 La comparaison entre les trois alliances révèle des similitudes dans leur composition, leur organisation et leurs réseaux. L'évolution des compagnies membres des alliances entre 1995 et 2005 suggère que des stratégies ont été mises en oeuvre pour réorganiser et coordonner leurs réseaux, bien qu'aucune preuve formelle ne permette de le confirmer 
complètement. Les alliances et les compagnies qui les composent communiquent très peu d'informations sur ce sujet sensible commercialement. On peut penser que, s'il n'est pas certain qu'il existe une véritable stratégie interne des alliances, il y a au moins dans le chef de leurs membres une vision cohérente, qui tend à créer un réseau intégré compétitif et qui n'est pas le résultat de la simple addition de réseaux de compagnies indépendantes.

Les alliances semblent être le fruit d'une stratégie de défense des compagnies aériennes dans un contexte de concurrence accrue plutôt qu'une structure permettant de conquérir de nouveaux marchés. L'offre des compagnies membres des alliances en 2005 a en effet augmenté moins rapidement que celle du transport aérien en général; les compagnies ont recentré leur offre sur quelques liaisons et ont supprimé plus de liaisons qu'elles n'en n'ont créées. Les sièges des alliances se sont concentrés sur les liaisons interrégionales, où la concurrence est plus faible et les profits plus élevés, alors que dans les sièges sur les vols intérieurs aux régions, où les compagnies low cost sont présentes et où la concurrence est forte, l'offre a stagné ou diminué. Cette stratégie de défense est surtout vraie pour les grandes compagnies, alors que les plus petites compagnies, principalement européennes, ont bénéficié d'une très forte croissance entre 1995 et 2005 sans doute grâce à la notoriété plus grande dont elles bénéficient dans leur réseau.

Le transport aérien conditionne en partie la localisation des activités économiques et touristiques dans le monde. Dans ce contexte, le recentrage des alliances sur les plus importantes liaisons entre les régions les plus riches et dans les principaux aéroports des plus grandes villes renforce le rôle des zones métropolitaines centrales et de ces pays au détriment des autres régions moins bien desservies. Cette tendance à la concentration semble ne pas devoir s'inverser, au moins pour les liaisons internationales, en raison des coûts du transport long-courrier et de la nécessité de remplissage des appareils. Cependant, la situation au niveau régional est plus contrastée: si on observe une concentration des activités à l'intérieur des alliances, les compagnies low cost se développent de leur côté à partir d'aéroports secondaires, à l'encontre de cette logique. Par ailleurs, le système de coopération et non de fusion, caractéristique de la concentration du secteur aérien, ménage la possibilité pour les compagnies d'établir des accords bilatéraux en dehors du réseau de leur alliance et de la quitter. Cette relative souplesse permet d'offrir aux compagnies un cadre pour résister aux aléas du développement de l'économie monde, mais constitue peut-être aussi une opportunité pour les alliances de se développer de manière indirecte dans des secteurs qu'elles ne couvrent pas de manière principale.

L'évolution et les stratégies des alliances de compagnies aériennes sont dictées par des impératifs économiques et politiques. Malgré la libéralisation progressive, il n'existe pas encore de véritables consolidations et fusions internationales de compagnies aériennes, à l'inverse de nombreux autres secteurs d'activités mondialisés, tels que les banques, les télécommunications, l'agroalimentaire..., ce qui fait une particularité du transport aérien. L'étude des alliances du point de vue géographique illustre le lien fort entre le trafic aérien et l'économie mondiale. Les modalités de l'éventuelle intégration des régions actuellement exclues des zones couvertes par les alliances et les compagnies qui les couvrent, pourraient être un sujet d'étude utile. 


\section{BIBLIOGRAPHIE}

AGUSDINATA B., DE KEIN W. (2002), « The Dynamics of airline alliances », Journal of Air Transport

Management, 8, pp. 201-211.

BEAVERSTOCK V., SMITH R.G., TAYLOR P.J. (1999), « A Roster of World Cities », Cities, 16 (6).

BIPLAN P. (2004), « Les compagnies aériennes entre la nation et la mondialisation », Hérodote, 114, pp. 57-70.

DENNIS N. (2000), « Scheduling issues and network strategies for international airline alliances », Journal of Air Transport Management, 6, pp. 75-85.

DENNIS N. (2005), « Industry consolidation and future airline network structures in Europe », Journal of Air Transport Management, 11, pp. 175-183.

DENTON N., DENNIS N. (2000), « Airline franchising in Europe : benefits and disbenefits to airlines and customers ", Journal of Air Transport Management, 6, pp. 179-190.

GUDMUNDSSON S. V., RHOADES D. L. (2001), « Airline alliance survival analysis : typology, strategy and duration », Transport Policy, 8, pp. 209-218.

GUDMUNDSSON S. V., DE BOER E. R., LECHNER C. (2002), « Integrating frequent flyer programs in multilateral airline alliances ", Journal of Air Transport Management, 8, pp. 409-417.

HANLON P. ( $2^{\text {nd }}$ ed., 1999), Global airlines : competition in a transnational industry, Butteworth Heinemann, Oxford.

KLEYMANN B. (2002), The development of multilateral alliances-the case of airline industry, Doctoral thesis, Helsinki School of Economics, Helsinki, Finland.

LACOSTE Y. (2004), « Aviation et géopolitique : les projections de puissance », Hérodote, 114, pp. 5-16.

LEE D. (2003), « Concentration and price trends in the US domestic airline industry : 1990-2000 », Journal of Air Transport Management, 9, pp. 91-101.

MERLIN P. (2000), Le transport aérien, La documentation française, Paris.

ORGANISATION MONDIALE DU COMMERCE (2003), « Le commerce international des services de transport aérien : évolution récente et questions de politique ", in OMC, Rapport sur le commerce mondial 2005, pp. 235-290.

OUM T. H., CHUNYAN Y., ANMING Z. (2001), « Global airline alliances : international regulatory issues », Journal of Air Transport Management, 7, pp. 57-62.

STRALE M. (2006), Géographie et stratégie des alliances de compagnies aériennes, mémoire de fin d'études en Sciences géographiques, Université libre de Bruxelles, inédit.

VOWLES T. M. (2000), « The geographic effects of US airline alliances », Journal of Transport Geography, 8, pp. 277-285.

WEBER M., DINWOODIE J. (2000), « Fifth freedoms and airline alliance. The role of fifth freedom traffic in an understanding of airline alliances ", Journal of Air Transport Management, 6, pp. 51-60. 


\section{NOTES}

1. Air Transport World, 31/05/2006, "Evolving Aer Lingus Confirms Oneworld departure ", Buyck, C.

2. La situation a évolué depuis 2005, avec l'entrée de South African Airways dans Star Alliance en 2006, reflet de l'insertion bien meilleure de l'Afrique du sud dans l'économie mondiale que celle du reste du continent africain.

3. Oneworld, http://www.oneworld.com/pressroom/releases/releases.cfm, 04/06/2006, "Japan Airlines to join oneworld alliance early next year ».

4. Skyteam, http://www.skyteam.com/FR/ aboutSkyteam/pressCenter/press.jsp, 28/06/2006, « Skyteam signe un accord avec China Southern Airlines ».

\section{RÉSUMÉS}

Les alliances de compagnies aériennes, phénomène récent dans le transport aérien, sont en plein développement. Cet article établit la géographie mondiale des alliances en 2005, à différentes échelles (régions, pays, aéroports), et leur évolution depuis 1995. Les trois alliances actuelles sont composées de compagnies issues en grande majorité des pays occidentaux et leur offre s'organise dans et entre les régions du centre de l'économie monde. Cette situation est le résultat d'une concentration et d'une réorganisation de l'offre des compagnies membres des alliances en réponse aux impératifs économiques de la mondialisation.

The airlines alliances, recent phenomenon in air transport, are under development. This article establishes the world geography of alliances in 2005, on various scales (country, regions, airports), and their evolution since 1995. The three main current alliances are made up of companies resulting in great majority from the developed countries and their offer is focused on the centre of the world economy. This situation is the result of a concentration and a reorganization of the offer of the alliance's members companies in response to the urgent economic requirements of globalization.

\section{INDEX}

Mots-clés : géographie des transports, transport aérien, alliances, mondialisation

Keywords : transport geography, air transport, airline alliances, globalization

\section{AUTEUR}

\section{MATHIEU STRALE}

Université Libre de Bruxelles, IGEAT (Institut de Gestion de l'Environnement et d'Aménagement du Territoire), mstrale@ulb.ac.be 